Please quote as: Sandner, U.; Leimeister, J. M. \& Krcmar, H. (2006): Business potentials of ubiquitous computing. In: Managing Development and Application of Digital Technologies. Verlag/Publisher: Springer, Erscheinungsjahr/Year: 2006. Seiten/Pages: 277-292. 


\section{Business Potentials of Ubiquitous Computing}

Uwe Sandner, Jan Marco Leimeister, Helmut Krcmar

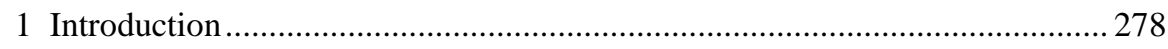

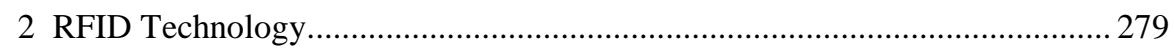

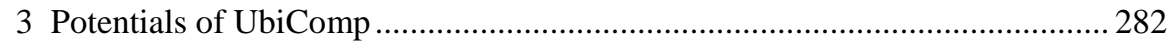

3.1 RFID as Enabler for More Efficient Identification Technology ................ 283

3.2 RFID as Enabler for the Real-time Enterprise ............................................ 286

3.3 RFID as Enabler for the Internet of Things .............................................. 287

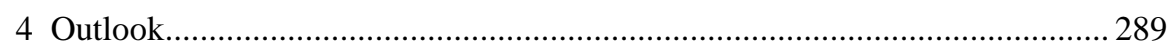

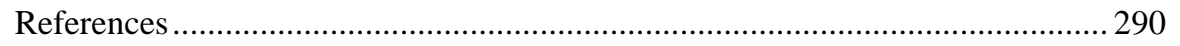




\section{Introduction}

The term ubiquitous computing was coined by Weiser (1991) and focuses on the increasing number of computing devices that are surrounding humans in everyday life. Physical objects, information and processes merge providing economical, social and individual benefits, such as new products, services, and business models, increased security and safety, and higher convenience for consumers. In UbiComp people will no longer be aware of their usage of IT systems since these have "disappeared" and become part of everyday products, so called Smart Devices (e.g. an intelligent fridge ordering automatically goods and services on demand, etc.) Today, UbiComp receives attention under the label Radio Frequency Identification (RFID).

RFID is, first of all, a technology that allows wireless communication and identification building on radio waves between objects. Secondly RFID means a system that includes not only the communication interface but hardware and software components that reach from physical infrastructure to applications, and thirdly, it enables some of the concepts that are necessary for building UbiComp systems. RFID systems allow the automatic data collection caused by real-world events and therefore the automisation of many manual data collection activities in and between companies (e.g. real-time monitoring of goods, etc.) The full exploitation of the potentials is by far not yet reached today, the full impact is still to come (Sheffi 2004). Today, automotive, manufacturing, pharmaceuticals, healthcare, and logistics are among the most important industries for RFID usage (Furness 2005; Strassner and Fleisch 2005; Strassner and Fleisch 2002; Fleisch and Dierkes 2003). This chapter gives an overview about potentials and challenges of ubiquitous computing (UbiComp) for business management. The objective is to introduce applications and usage scenarios that might be supported by UbiComp. The analysis starts with a definition of an UbiComp system and its components. Potentials of UbiComp are identified categorized into three major areas: as an identification and information technology, as an enabler for improving processes and decisions in a real-time enterprise, and as a driver for the long-term vision of the "internet of things". For each area challenges and issues are identified. In concluding we highlight aspects managers should consider when thinking about their future IT landscapes and business opportunities. 


\section{RFID Technology}

The basic technology in a RFID system consists of an information and communication technology (hardware) layer including objects, tags, readers, sensors, and actuators, and an information system layer including middleware, applications, and interfaces as software components (Fig. 1).

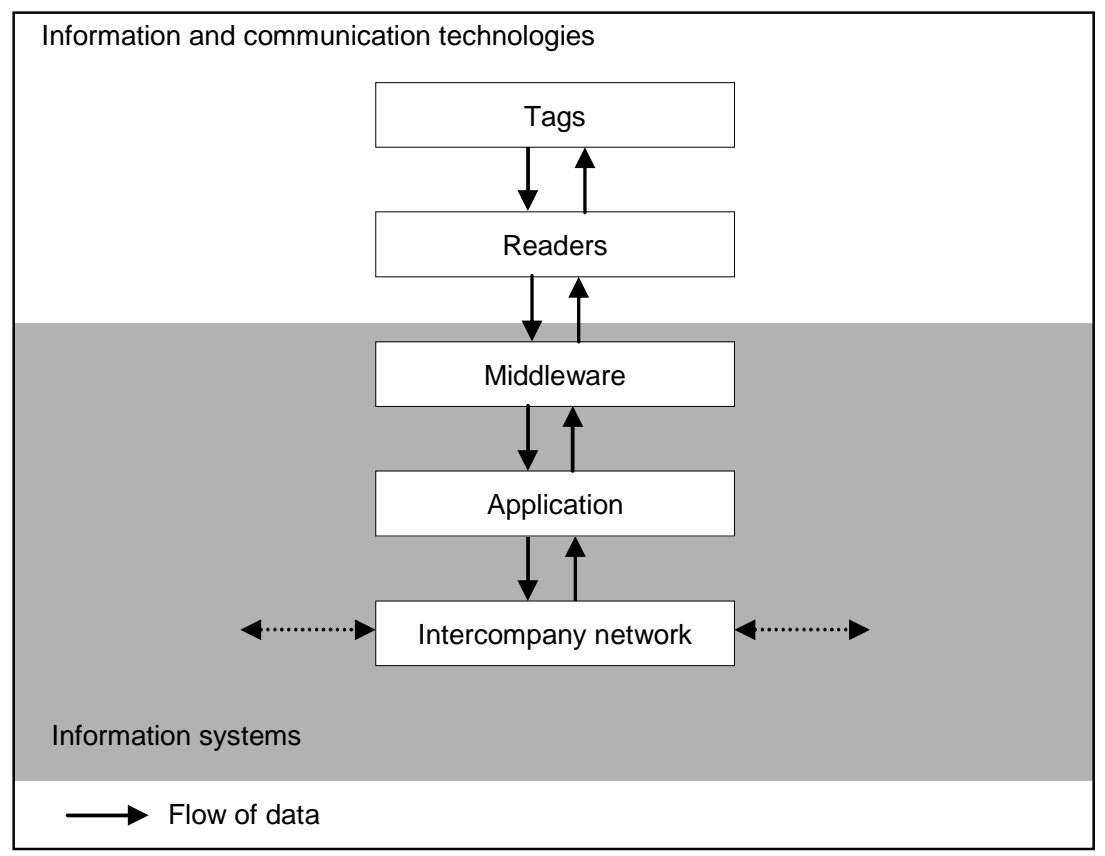

Fig. 1. UbiComp system

An object is a physical item, for example a good, a machine, an asset, a resource, a container, a vehicle, a pallet, or a living being, such as an animal or a person, that is equipped with a tag. To identify persons, tags may be attached to an object the person possesses like a garment, a passport, or a key. All objects in the RFID-system covering a specific sector of the "physical world" have a tag.

A tag is a piece of hardware attached to an object and able to communicate with a reader. Tags store data and the most important data is a unique ID for the object. They may possess additional storage capacity and interfaces to sensors. Tags are attached to the objects for enabling their automated identification and they come in several flavors which differ in size, price and technical capabilities. Among the most important tag characteristics are the need for power supply (i.e. passive tags need to internal power 
supply), communication range, capacity of data storage, ability to perform calculations and logic, and interfaces to sensors. To protect the tag from its environment different bodies are used, like glass or plastic coils or the tag is applied on paper or foil or directly printed on the object. The tags communicate with readers on a wireless radio interface. Since the physical characteristics as the materials (metal, fluid) of the object might influence the communication, and in many situations objects are hidden beneath other objects, packed together on pallets or in containers or shielded by other objects, the place of the tag must be selected properly.

A reader is a device that communicates on one side with tags, on the other side with a backend system. Readers can be split into several components again. The reader has first, a wireless communication interface to the tags, second, a communication and processing interface to the middleware layers, and, third, a reader management component. The communication interface to the tags must ensure that events in the physical world like the change of the status of an object are received correctly. This raises data transmission challenges, especially in areas where several readers are used. Communication bandwidth is limited and when several readers want to send and receive information at the same time, this causes collisions. The same problem can occur when there are several tags which want to send information. Especially when tags are moving and are only for a limited time in the reading range of a reader, the correct recognition of physical events becomes difficult. Basically, this limitations lead to the phenomenon that physical changes of objects are not recognized properly in the system consisting of the tag, the radio communication interface and the reader. A problem is that changes (such as the movement of an object) are completely missed and not grabbed by the reader. Strategies to alleviate this problem include the coordination between several readers to reduce collision and the prioritization which changes and which objects are most relevant.

The second component of the reader is the interface to the middleware, where raw data events are transformed and transmitted to middleware servers. Some of the functions like prioritization, cleansing, filtering and aggregation can be done in the reader, reducing the amount of data to be communicated to the middleware. This is especially relevant if reader and middleware communicate over channels with limited bandwidth or discontinuous connectivity, for example with mobile readers and mobile communication networks. The reader management component becomes relevant in usage scenarios where there are many devices, for example at a production site. To reduce the effort to administrate and check the reader hardware which might be placed in difficult to reach locations and to ease collaboration between several reader devices. With ongoing technology 
development, the capabilities of readers will grow and shift more functions from the middleware to the readers.

The middleware is a component that performs data processing tasks like aggregation and filtering and makes the raw data from the reader usable by applications. A lot of raw physical events, which are not relevant for any application, are gathered by the reader, so strategies for distributing and forwarding only relevant data are necessary. Data cleansing, filtering and aggregation are also necessary. The middleware also takes part of the management of the infrastructure and should hide problems on the communication layer (Krishna and Husak 2005; Floerkemeier and Lampe 2005).

The application system connects the UbiComp applications with other IT systems in an organization, for example for supply chain management and resource planning. It includes functions to support management decisions and to control and monitor processes.

While the application system focuses on the single enterprise, the intercompany system connects several organizations across the value chain. The application system also includes infrastructure and services that deal with information exchange, interoperability and directories.

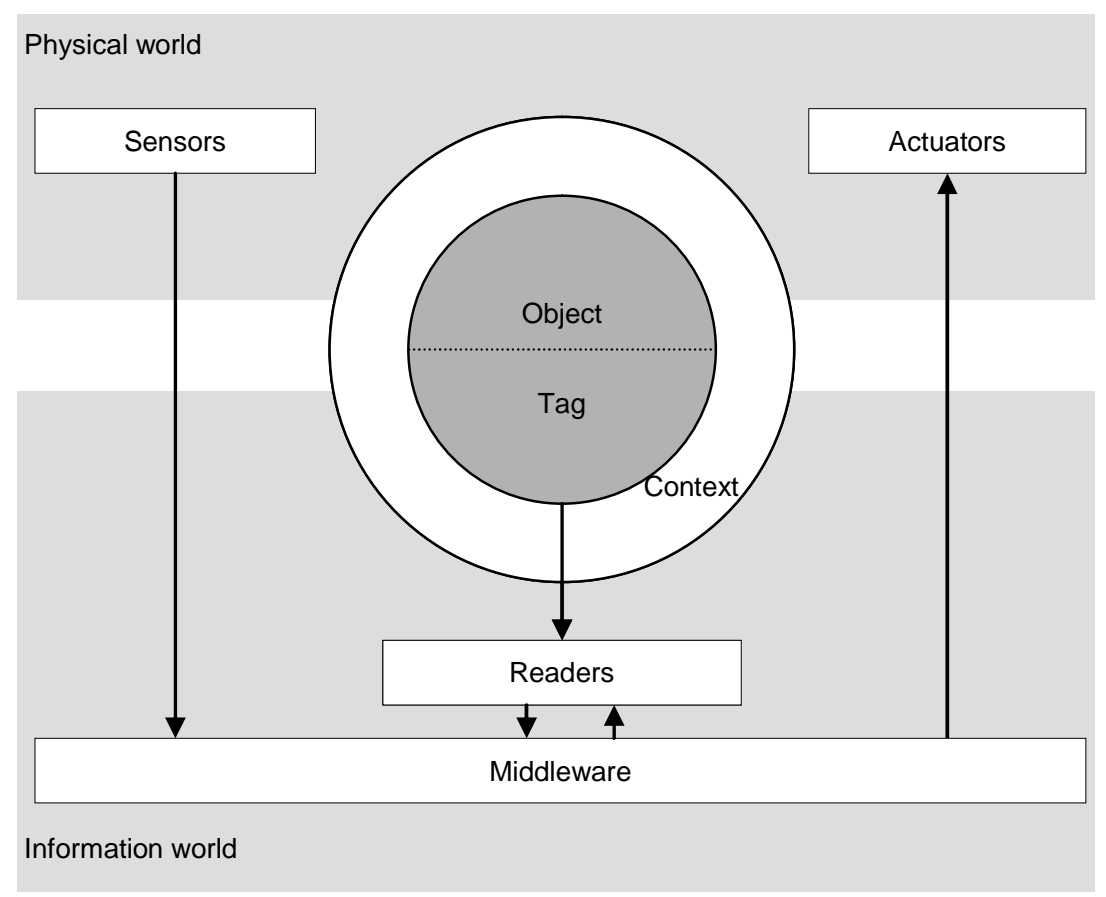

Fig. 2. UbiComp as bridge between physical and information world 
Context is information about an object including the unique ID, sensor data, and information gathered or created during the lifetime of the object. Context information may be stored on the tag or by the backend system (Finkenzeller 2002).

A sensor is a piece of hardware that is able to gather information about its environment such as position, temperature, pressure, brightness, or moist. Sensors might be placed at the object and communicate directly with tag or might be external source that are connected to the UbiComp system (Dongwon et al. 2005).

An actuator is a device that influences its environment physically, for example a machine, a robot, or a display. It might be controlled by an UbiComp system to adapt the environment and process actions for objects.

UbiComp systems bridge the gap between the physical world and the information world, in this case by merging physical world objects with tags (Fig.2). Thus it becomes possible to process automatically an object's context. The middleware does not only provide access to via readers to this artifact, but also to sensors and actuators.

To make information accessible to humans and maje the potentials of UbiComp usable for improved convenience, productivity and efficiency, adequate human-machine interfaces must be designed. They have to present information and capture input in a usable way (Nagumo 2002; Raskar et al. 2005).

\section{Potentials of UbiComp}

The potentials of UbiComp could be achieved in a three step roadmap (Fig. 3).

- First, RFID can become a driver for efficiency through improved performance at reduced prices for existing processes (this e.g. is the current state of mind for RFID usage in retail industry).

- Second, RFID might increase integration and efficiency through improved new processes and decision structures.

- And third, RFID might enable a paradigm shift leading to the Internet of Things, where new products and services lead to new business models and higher quality of life for everyone. 

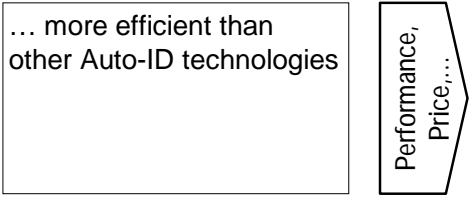

Identify with increased efficiency

Identify objects on a unique per item level

Get context of objects
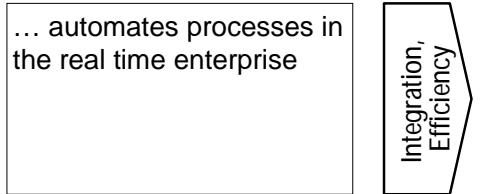

Better decisions by managers Improved integration of processes and partners
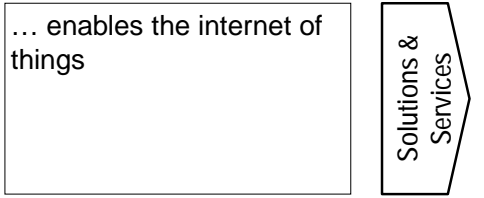

Self management of objects New services and products Improved quality of life

Fig. 3. Potentials of UbiComp

\subsection{RFID as Enabler for More Efficient Identification Technology}

In this stage RFID serves primarily as identification and information technology. The potentials and benefits of UbiComp lie in more efficient processes (e.g. in automated goods receipts in retail or manufacturing, etc.), with higher performance at cheaper prices. RFID systems combined with optimized processes allow better management of information in companies and organizations and to solve problems and shortcomings of existing processes.

\subsubsection{Potentials}

\section{Identify with increased efficiency}

Since costs for identification are reduced, identification can be used more often and in finer granularity throughout the process chain, where first applications are seen in logistics and supply chain management (Kinsella 2003; Twist 2005). This leads to decreased cost of identification of flows of goods, since UbiComp makes the identification of objects faster, easier and more error prone compared to traditional identification methods as barcodes or labels. Readers do not need a line of sight to the objects, which allows the scanning of objects packed together or stored in containers. 
Tags are more resistant against dirt, damage or other environmental influences. Identification can be automated without human interaction and thus human mistakes. Furthermore it is faster since many objects can be recognized at the same time. This can for example be used in logistics, where a large number of objects have to be processed in short time.

\section{Identify unique objects on an item level}

Objects are identified not only on a generic class level, but each item has a unique electronic identity. Customers and consumers can be protected against fraud (e.g. by storing the unique identification number and the way of the good through the sales channel) and can be sure that the good they own does not only look like the one they wanted but was originally manufactured by a certain company. This is not only relevant at product piracy in industries with high value brand name items, but also for spare parts and products where a possible reduction in quality or changed technical characteristics can cause damage. Safety and security are increased, when objects are easier to distinguish, for example in health applications, where correct drugs and treatments should be applied to the correct patient, or for homeland security and access control.

\section{Get context of objects}

The identification of objects always comes with at least one type of context data: the information that the object was in the proximity of a reader (which has a position) at a certain point of time. This context can be used to make it easier to prevent theft (e.g. by detecting when a good leaves a certain area or by verifying the original owner), to locate items in a warehouse, in production facilities or container management or on promises for logistics. When the identification information is merged with sensor data and these data are stored historically, objects can be traced. Single items of a production series are in many cases difficult to distinguish when they arrive at the customer. When problems are discovered that occurred earlier in the process chain (e.g. hidden defects) it is a complex and expensive task to trace all products and repair the defect. For some items it is also important that an item was not processed in certain locations, by certain persons or organizations because negative influence or the alteration of the item could lead to security of safety problems. When an object can be identified uniquely, this can be used to access information related to this single object and does process items not only based upon a general object class and its general characteristics. In manufacturing and logistics a higher flexibility and granularity can be reached. 


\subsubsection{Challenges}

\section{Reliable hardware level}

According to experience reports on current implementations of RFID, the reliability of processes on a hardware layer is a challenge. Defective tags may lead to objects invisible to a system, as limited bandwidth, errors occurring on the air interface between readers and tags, interference by external sources or unfavorable environmental conditions. The $100 \%$ prevention of such failures might be impossible or cause significant costs.

\section{Availability \& accessibility of information}

When information and context is stored in a system which is interconnected by a communication network, an entity which requests data is confronted with problems of availability, consistency and security. Information might be stored at several physical or logical locations and might be replicated to increase efficiency. Directories are necessary to find this storage location and transmission of data must be protected against tampering.

\section{Privacy and access control}

In many situations it must be assured that only a limited number of organizations are able to use information stored on the tag (identification) or information connected to the context of an object. This leads to concerns in regard of privacy but also to complex decision who can access information (Data $\mathrm{X}$ was collected by organization $\mathrm{Y}$ at time $\mathrm{T}$ and is stored at $\mathrm{Z}$ - who is allowed to access or change this data?) (Ohkubo et al. 2005; Peslak 2005).

\section{Precision and quality of information}

Context information coming from sensors and external sources is available in several degrees of precision. Especially for position information the quality of data might be more a probabilistic assumption and not sufficient to support certain applications. Applications must be able to cope with these diffuse data and be able to use other technologies where necessary.

\section{Standards}

Since hardware such as readers and tags come from different manufacturers, there should be standards to hide specialties of single implementations. This abstraction (e.g. of hardware types, communication protocals, data exchange formats) must not only cover technical details but also include standards to exchange information about context, its representation, and logic to control and manage hardware. 


\subsection{RFID as Enabler for the Real-time Enterprise}

UbiComp might enable managers and organisations to make faster and better decision and to better plan and control processes. This is the core of the vision of the the Real-Time Enterprise (RTE), a vision coined by Gartner Research and others. The fundamentally idea is that an enterprise can gather real-time information, allowing it to change its operational characteristics dynamically in response to changing external parameters and to generate new sources of revenue, avoid unnecessary business expenses and significantly improve business processes (Neil and Clark 2003). In a RTE managers can use the information about identification and context to manage their enterprises in real time and use resources more efficiently. On the other hand, control can be moved down from a central Enterprise Resource Planning system to the location much closer to the processes and questions involved, leading to new problem solving strategies.

\subsubsection{Potentials}

\section{Better decisions by managers}

UbiComp provides managers and consumers with more information and leverages better, more efficient and faster decisions (Smith and Mckeen 2005). The abundance of information does not come for free, since the representation and processing of data must be in a format that does not overstrain human abilities. Decisions must be supported in a proper way and the interface to users must be appropriate. For some items the process of the identification of the item and the provisioning of connected information (handling instructions, details about characteristics) is complex from a process view including human interaction. These processes can be simplified, for example in a shop floor environment where consumers receive details about a product or in a manufacturing process where workers receive handling instructions.

\section{Improved integration of processes and partners}

UbiComp can improve the integration of processes and partners in enterprises by providing better and more detailed information about the status of goods in the supply and the production chain (Van Nunen and Zuidwijk 2004; Krikke et al. 2004). Management can react faster to challenges and changing internal and external requirements which allows providing additional value to customers and taking market changes into account. For outbound logistics and after sales services higher service levels can be reached and sold. 


\subsubsection{Challenges}

\section{Information retrieval}

More information does not automatically lead to better decisions when the excess of data is not analyzed and aggregated in a way managers can understand and use. This challenge has been addressed for more than two decades in the context of management information systems (MIS) and many challenges (e.g. as to adequate task-technology fit of such MIS or information retrieval needs of managers, etc.) have to be taken into consideration. The design of human-machine interfaces and the representation of relevant information is a challenge that must be solved before UbiComp can leverage all potentials.

\section{Middleware and applications}

The middleware in UbiComp systems is a key component to connect to hardware from several manufacturers and information systems in companies. Due to the diversity of existing products and legacy systems already in place the flexibility and ability to integrate those solutions is critical. Application systems must not only be able to analyze and monitor the surplus of information made available by UbiComp, but also support control structure and processes. Furthermore, the amount of generated data increases which presents challenges to the communication and information system infrastructure. Necessary up front investments to make middleware and applications available have to be weighted out against benefits.

\subsection{RFID as Enabler for the Internet of Things}

UbiComp can not only lead to more distributed processes in companies, but also allows a stronger interconnection of organizations in the value chain: From manufacturers, suppliers, retailers, service providers, up to customers and consumers. Companies can keep an information link to every physical object they processed, shipped, manufactured or used at any time which allows new business models shifting the focus from the single specific step in the value chain to a more holistic view where solutions and services become more prominent (Fleisch 2001).

\subsubsection{Potentials}

\section{Self management of objects}

The vision of the internet of things, where every item as simple as a light bulb (Gershenfeld et al. 2004) is connected to the internet, leads to the question which form of problem solving (centralized vs. distributed) and 
control (company centric vs. holistic) does provide benefits for certain tasks and physical goods and how business models can realize these benefits. Processes are not planned centrally, but autonomous, decentralized "smart" units solve problems on their own. The shift of problem solving from a central location to multiple single processes is also an area of research in other fields, like organic computing.

Items can report information about themselves (identification), their status and their environment with the help of sensors, be able to communicate with other objects, possess computational power to process data, and influence their surrounding with actuators. Given that many objects are smart they form a swarm which leads to a distributed, decentralized system. In an enterprise context, this idea can be broken down into the vision of the virtual enterprise where all resources (people, goods, products, assets) and their related processes are monitored and controlled in real time.

This changes the location where information is stored and processed and where processes are changed. When a machine in the production line is recognized to cause problems or stops working, an alternative production setup can be discovered by objects and they can "decide" to use an alternative machine for the next step. Objects can use actuators to adapt their environment so it fits their needs, leading to self-healing processes.

\section{New services and business models}

Ubicomp enables companies to keep a connection to the products and goods manufactured or processed through the whole product lifecycle, even when the object is located at the premises of the customer. This allows completely new services and business models where solutions become more important. Not only do physical characteristics of a product define the benefit it delivers, but also connected and surrounding services. Furthermore, different pricing and billing models are possible, from price discrimination based on more information on customers to billing on a perusage base. Processes can be divided in more granular and individual steps, leading to finer measurement of the value contribution of single players and virtual organization forms (Banavar et al. 2005).

\section{Improved quality of life}

Although UbiComp is seen as a threat for privacy, it has the potentials to improve the quality of life for everyone. As a consumer, people receive more information about products leading to higher safety and security, for example in areas such as food or drugs. In health and emergency response applications, faster response times and fewer mistakes become feasible. The usage in homeland security plays an important role in countries that face the danger of terrorist attacks. This sector is becoming a major driver for research \& development on UbiComp technologies. 


\subsubsection{Challenges}

\section{Standards}

If the potentials of UbiComp should be exploited fully, an application must not only focus on single organizations but should be able to span across the whole value chain and integrate multiple players along the value chain such as suppliers and retailers. This needs first common standards and data exchange structures to allow sharing identification, information, and context about objects, but also interfaces to connect processes and decisions. Different solution providers and manufacturers as well on the information and communication technology layer as on the information systems need to make their solutions able to communicate with each other.

\section{Global infrastructure}

To allow the interconnection of enterprises and their UbiComp applications there is a need for a technical infrastructure that enables these interconnections. Similar structures are already present in the World Wide Web, for example the domain name service. Also, the organizational aspects of these structures (operation of the infrastructure, management of unique IDs, certification of players) have to be discussed, where for example EPCGlobal could play an important role.

\section{Acceptance and Privacy}

UbiComp provides vast opportunities for consumers, but also faces challenges in regard to privacy. Companies, lobbyists and governments have to inform and educate the public (Eckfeldt 2005; Günther and Spiekermann 2005 ) and inform about risks and privacy issues. At the same time, technical solutions must be found to allow people to control their privacy, to identify UbiComp technology in products they buy or use.

\section{Intelligent objects}

When objects should sense their environment, plan ahead, interact with other objects and decide, algorithms are requires which give objects these capabilities and intelligence. Research fields like organic computing, semantic modeling, swarm intelligence and agents can give important input to solve these problems (Brock et al. 2005; Bodendorf and Zimmermann 2005).

\section{Outlook}

UbiComp provides vast benefits in several areas, from replacing current identification technologies to having large impact on society and economy. 
In a first step, it can become an efficiency driver for optimizing existing processes and realize cost benefits. Afterwards it will allow rebuilding and automating processes to a higher degree than today by giving management the tools to decide better and faster. At the same time, control over processes will move from central application systems to the locus where problems arise and enable smart objects to shape their environment. Enterprises can integrate internal and external steps of the value chain to a larger extend, making economy more effective. With the help of a global infrastructure, the internet of things might have huge impact on economy and provide individual benefits to everyone.

But there are also challenges and risks from technical problems on the hardware layer, question on the algorithms of intelligence for objects, up to privacy issues in the social and legal framework. Standards and the question of integrating existing systems to make the vision possible also need further research.

Companies should keep in mind that the importance of RFID as a core technology of UbiComp is already rising, and cannot be ignored for much longer. It will not only be about replacing barcode, but will affect a lot more processes, products or services. They also should be well aware of the fact that RFID usage is not only a thing of their own choice, but can also be declared necessary or even compulsory on the part of business partners, as happened in the cases of Wal-Mart and Metro. It is therefore essential to keep watch of the RFID activities of close neighbours in the supply chain. Companies are well advised to concretize and substantiate their RFID visions in order to know their needs and possibilities and to develop an action plan they can refer to.

\section{References}

Banavar G, Black J, Cáceres R, Ebling M, Stern E, Kannry J (2005) Driving Long-term Value from Context-aware Computing. Information Systems Management, 22, 32-42

Bodendorf F, Zimmermann R (2005) Proactive Supply-Chain Event Management with Agent Technology. International Journal of Electronic Commerce, 9, 5789

Brock DL, Schuster EW, Allen SJ, Kar P (2005) An Introduction to Semantic Modeling for Logistical Systems. Journal of Business Logistics, 26, 97-117

Dongwon J, Young-Gab K, Hoh I (2005) New RFID System Architectures Supporting Situation Awareness under Ubiquitous Environments. Journal of Computer Science, 1, 114-120

Eckfeldt B (2005) What Does RFID Do for the Consumer? Communications of the ACM, 48, 77-79 
Finkenzeller K (2002) RFID-Handbuch, München, Wien, Carl Hanser Verlag Fleisch E (2001) Business perspectives on Ubiquitous Computing.

Fleisch E, Dierkes M (2003) Ubiquitous Computing aus betriebswirtschaftlicher Sicht. Wirtschaftsinformatik, 45, 611-620

Floerkemeier C, Lampe M (2005) RFID middleware design: addressing application requirements and RFID constraints. Proceedings of the 2005 joint conference on Smart objects and ambient intelligence. Grenoble, France, ACM Press

Furness V (2005) The RFID Market Outlook - New applications, best practices and future profit opportunities. Business Insights

Gershenfeld N, Krikorian R, Cohen D (2004) The Internet of Things. Scientific American, 291, 76

Günther O, Spiekermann S (2005) RFID and the Perception of Control: The Consumers View. Communications of the ACM, 48, 73-76

Kinsella B (2003) THE Wal-Mart FACTOR. Industrial Engineer: IE. Institute of Industrial Engineers

Krikke H, le Blanc I, van de Velde S (2004) Product Modularity and the Design of Closed-Loop Supply Chains. California Management Review, 46, 23-39

Krishna P, Husak D (2005) Simple Lightweight RFID Reader Protocol.

Nagumo T (2002) Innovative Business Models in the Era of Ubiquitous Networks. NRI Papers. Nomura Research Institute

Neil D, Clark W (2003) The Real-Time Enterprise and the Network. Gartner

Ohkubo M, Suzuki K, Kinoshita S (2005) RFID Privacy Issues and Technical Challenges. Communications of the ACM, 48, 66-71

Peslak AR (2005) An Ethical Exploration of Privacy and Radio Frequency Identification. Journal of Business Ethics, 59, 327-345

Raskar R, Beardsley P, Dietz P, van Baar J (2005) Photosensing Wireless Tags for Geometric Procedures. Communications of the ACM, 48, 46-51

Sheffi Y (2004) RFID and the Innovation Cycle. International Journal of Logistics Management. International Logistics Research Institute

Smith HA, McKeen JD (2005) Developments in Practice XV: Information Delivery: IT's Evolving Role. Communications of AIS, 2005, 197-210

Strasser M, Fleisch E (2005) Innovationspotenzial von RFID für das SupplyChain-Management. Wirtschaftsinformatik, 47, 45-54

Strassner M, Fleisch E (2002) Segment Evaluation Automotive - Applications of RFID Technology.

Twist DC (2005) The impact of radio frequency identification on supply chain facilities. Journal of Facilities Management, 3, 226-239

van Nunen JAEE, Zuidwijk RA (2004) E-Enabled Closed-Loop Supply Chains. California Management Review, 46, 40-54

Weiser M (1991) The Computer for the 21st Century. Scientific American, 265, 94 
